\title{
Size distribution of SNRs and the ISM
}

\author{
Abdul I. Asvarov \\ Institute of Physics, Baku AZ1143, Azerbaijan Republic. \\ email: asvarov@physics.ab.az
}

\begin{abstract}
Based on the very general assumptions on evolution, we have modeled the statistics of SNRs evolving at various initial and environmental conditions. The method is applied to M33, for which the value of the filling factor of the hot phase of the ISM is estimated to be $\sim 10 \%$.
\end{abstract}

Keywords. SNRs, (ISM:) supernova remnants, galaxies: M33

The large scatter in the observational properties of SNRs can be explained partly by the large differences in the initial parameters of SNe and partly by the large differences in the characteristics of the ISM (density, pressure, etc.). Moreover, it is unclear what fraction of really existing SNRs we observe. To answer these questions we have constructed a simple model describing the statistics of SNRs, occurring with randomly distributed initial and environmental parameters. In our model, the density of the ISM is the most important parameter as its value changes over a wide range - for this parameter we have used three-phase model of McKee \& Ostriker. It is obtained, that the filling factor of the hot phase is the main parameter which determines the shape of the size distribution of SNRs. For the comparision with the observational data the catalogue of SNRs in M33 of Long et al.(2010) is used. In the list of X-Ray SNRs we select the subset of objects with hardness ratio $H \sim-0.5$ as the subset which has a high degree of completeness.
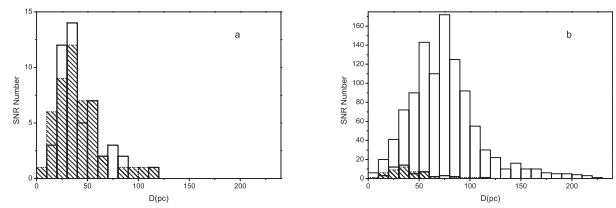

Figure 1. Distributions of modeled (solid lines) and observed (dotted lines) in M33 SNRs with $\mathrm{H}=-0.5 \pm 0.2$ (a) and their position in the distribution of all modeled SNRs in M33 (b).

An excellent coincidence between distributions of this subset of SNRs and the subset of modeled SNRs with the same value of $H$ was reached at the filling-factor of the hot phase of the ISM in M33 of 10\% (Fig.1a). The position of this subset of SNRs in the general distribution of generated SNRs (Fig.2a) shows that the observed SNRs represents very small fraction of really existing SNRs. The SNe birthrate for M33 is estimated to be $120-150 \mathrm{yr}^{-1}$, the full number of unseen but with Mach number $\geqslant 2$ estimated $\geqslant 1200$ (this value depends on the total pressure of ISM).

This work was supported by the Science Development Foundation under the President of the Republic of Azerbaijan Grant No EIF-2010-1(1)-40/05-M-23.

\section{Reference}

Long, K. S., Blair, W. P., Winkler, P. F., et al. 2010, ApJS 187, 495 